\title{
Development of a 13 kW Hall Thruster Propulsion System Performance Model for AEPS
}

\author{
Steven Stanley ${ }^{1}$, May Allen ${ }^{2}$, Keith Goodfellow ${ }^{3}$ \\ Aerojet Rocketdyne, 11411 139th PL NE, Redmond, WA 98052-2025 \\ Gilbert Chew ${ }^{4}$ and Ryan Rapetti ${ }^{5}$ \\ Aerojet Rocketdyne, 2001 Aerojet Road, Rancho Cordova, CA 95742-6418 \\ Todd Tofil ${ }^{6}$ and Dan Herman ${ }^{7}$ \\ NASA/Glenn Research Center, 21000 Brookpark Road, Cleveland, OH 44135-3127 \\ Jerry Jackson ${ }^{8}$ \\ Aerojet Rocketdyne, 8900 De Soto Ave, Canoga Park, CA 91309 \\ Roger Myers ${ }^{9}$ \\ Consultant, R Myers Consulting, LLC, Woodinville, WA 98072
}

The Advanced Electric Propulsion System (AEPS) program will develop a flight 13kW Hall thruster propulsion system based on NASA's HERMeS thruster. The AEPS system includes the Hall Thruster (HT), the Power Processing Unit (PPU) and the Xenon Flow Controller (XFC). These three primary components must operate together to ensure that the system generates the required combinations of thrust and specific impulse at the required system efficiencies for the desired system lifetime. At the highest level, the AEPS system will be integrated into the spacecraft and will receive power, propellant, and commands from the spacecraft. Power and propellant flow rates will be determined by the throttle set points commanded by the spacecraft. Within the system, the major control loop is between the mass flow rate and thruster current, with time-dependencies required to handle all expected transients, and additional, much slower interactions between the thruster and cathode temperatures, flow controller and PPU. The internal system interactions generally occur on shorter timescales than the spacecraft interactions, though coordination with the spacecraft commands and telemetry will be at the higher frequency. The AEPS system performance model is designed to account for all these interactions in a way that allows evaluation of the sensitivity of the system to expected changes over the planned mission as well as to assess the impacts of normal component and assembly variability during the production phase of the program. This paper describes the initial efforts toward the system performance model development, correlation to NASA test data, and how the model will be used to evaluate the critical internal and external interactions. The results will ensure the component requirements do not unnecessarily drive the system cost or overly constrain the development

\footnotetext{
${ }^{1}$ System Performance Analysis Engineering Specialist, Senior AIAA Member

${ }^{2}$ Chief Engineer for AEPS

${ }^{3}$ Chief Engineer, Senior AIAA Member

${ }^{4}$ Control System Analysis Engineering Specialist, Senior AIAA Member

${ }^{5}$ Control System Analysis Engineering Specialist

${ }^{6}$ NASA EP Development PM and Advanced Electric Propulsion System (AEPS) contract COR

${ }^{7}$ NASA Solar Electric Propulsion (SEP) Project - Ion Propulsion Subsystem Lead, AIAA Associate Fellow

${ }^{8}$ Program Manager for AEPS, AIAA Member

${ }^{9}$ Consultant, AIAA Fellow
}

American Institute of Aeronautics and Astronautics 
program. Finally, the model will be available to quickly troubleshoot any future unforeseen development challenges.

\section{Nomenclature}

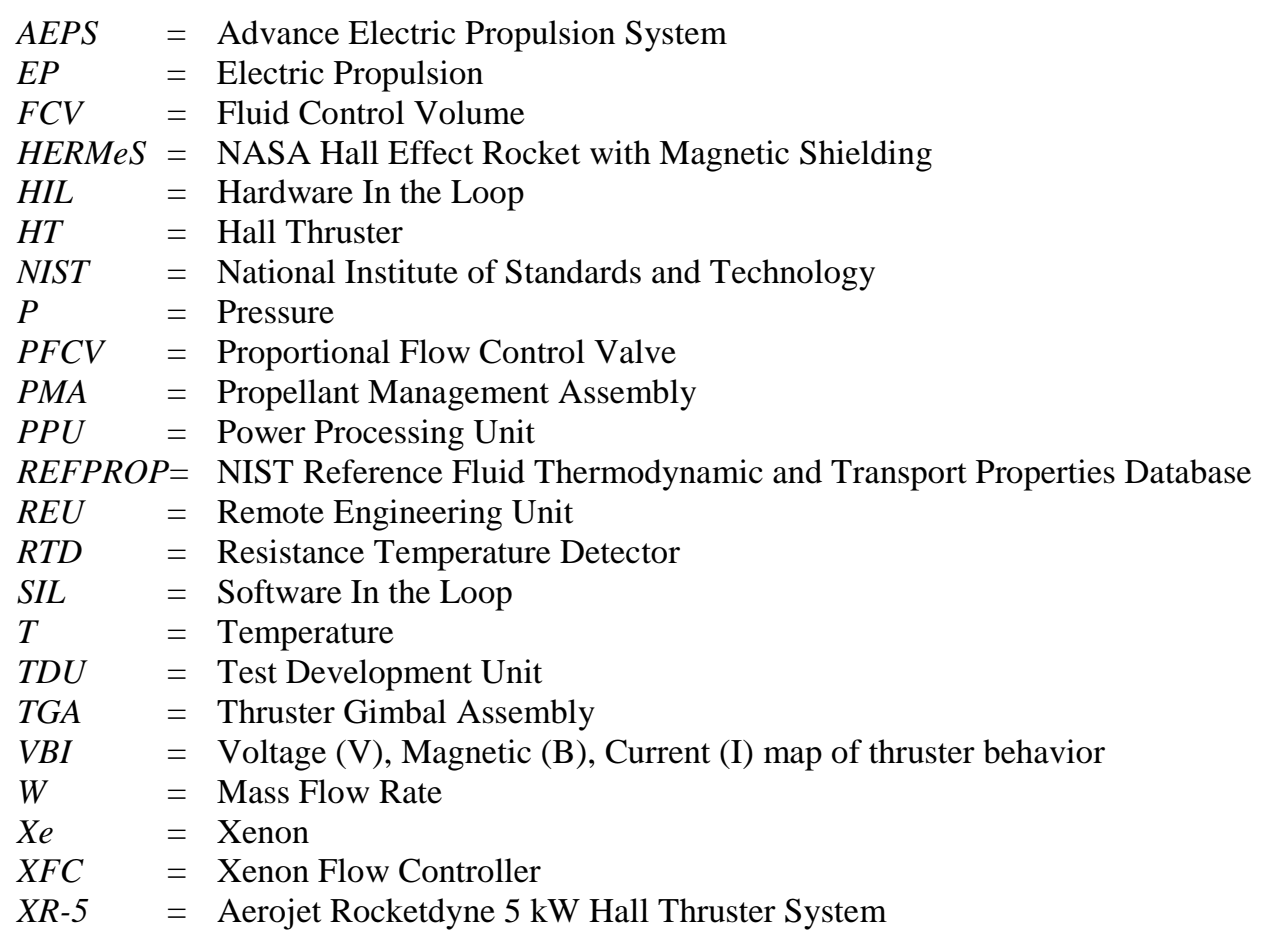

\section{Introduction}

$\mathrm{T}$

The significant advances in Hall thruster (HT) lifetime demonstrated by Aerojet Rocketdyne's flight XR-5 Hall Thruster and extended by NASA's development of magnetic shielding models demonstrated in the NASA HERMeS system program have enabled the use of high power HT systems for long-duration missions. ${ }^{1,2}$ Additionally, the recent discovery of a slightly modified system electrical configuration, with the thruster cathode electrically tied to the thruster chassis, has significantly improved the ability to properly ground-test higher power Hall thrusters. ${ }^{3}$ The AEPS program incorporates both of these improvements into the development of a flight $13 \mathrm{~kW}$ Hall thruster system, enabling the use of high power Hall thrusters for long duration NASA, DoD, and commercial missions. 
To ensure successful development of the AEPS flight system Aerojet Rocketdyne is developing a time dependent system performance model to address the interactions between components, design adjustments, production tolerances as well as the major interactions with the spacecraft. The program objective is to establish an EP string that can be integrated into a variety of spacecraft configurations for a range of Earth and deep space missions, so the system model must be written to accommodate a range of spacecraft interfaces. Figure 1 shows the notional interfaces between the AEPS (inside the dashed box in the

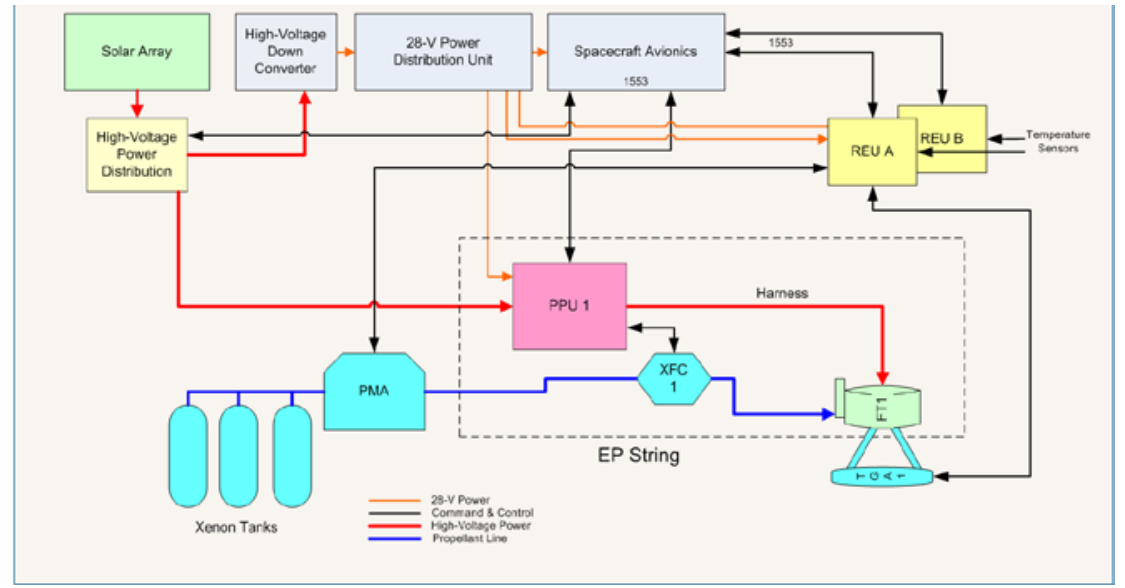

Figure 1. Notional Advanced Electric Propulsion System Integration with Spacecraft Bus Showing the Primary External and Internal Interfaces. figure) and a spacecraft system.

The system performance model will simulate how the system behaves both transiently and steady-state to help ensure that the thruster will operate over all mission phases and durations and is structured to allow easy updates to the spacecraft interfaces in order to support evaluations for a wide range of mission opportunities.

As shown in Figure 1, the AEPS EP String, denoted by the dashed line box, contains the Power Processing Unit (PPU), Xenon Flow Controller (XFC) and Hall Thruster (HT). It is also clear that the EP string interacts with the spacecraft in a number of ways. The PPU accepts high and low power inputs as well as the command interfaces including feedback telemetry. The XFC connects with the xenon propellant feed system and delivers propellant to the thruster (including the cathode), and has a fast feedback loop to the PPU controlled by way of the thruster current. Within the EP string, there are many more details than those shown in Figure 1. The internal systems and interdependencies are the focus of the system model and is a part of the development of AEPS. This paper summarizes the overall system development model approach, status, and the steps that will be taken to validate the code once completed.

\section{AEPS System Model Development}

The system model for AEPS is intended to be useful for development of the control methodology and the control system and provide understanding of the basic operational behavior of the full AEPS system including usage at the next higher level of integration. With that in mind, the system model has to inform on relational behavior between independent variables, but in order to be practical, the model cannot be computationally intensive. The balance between modeling the physics down to the atomic interactions within the plasmas and providing a simple response model is particularly challenging on AEPS due to the complex interactions within the plasmas, especially within the cathode. As a result of the unique challenges, the system model makes use of physics where practical but also uses data driven empirical approximations where the physics calculations are intensive. Initially, the model will capture the bulk of the interactions, on the fluid dynamic time scales, and will capture first-order plasma and electronic interactions. Phenomena occurring at much higher frequencies are not captured at the initial stage of development for this model.

For the model, a set of desired capabilities were established prior to beginning the model development. The intent was to outline a list of functions for the model to be capable of achieving and then if necessary reduce the features to improve the execution speed of the model to improve utility. One of the top goals was to have a practical model that would execute within an order of magnitude of real time, such that a real time minute of operation could be simulated in less than 10 minutes of execution time. At no time was the model intended for Software/Hardware In the Loop, SIL/HIL, testing, but that capability has not been explicitly ruled out.

One of the primary functions of the AEPS system model is to assist in the development of the control logic for the system. In order to develop the feedback and control loops for AEPS, the relationship between the mass flow rate and the current at a given voltage and magnetic strength has to be known or calculable. Since the Voltage-Magnet-Current 
(VBI) characteristic is rather complex, a single map approach is used to capture that relationship. With the main thruster flow known, it is possible to then develop the XFC flow control logic and the gains associated with it. Similarly, the cathode vs flow behavior dictates the cathode flow control behavior that is provided by the XFC.

The xenon flow functions of interest include accurately modeling the feed system response to valve movement and the conditions within the cathode and anode. With this basic functionality, the feedback controls can be developed for commanding flow rate as needed. Therefore, pressure drops throughout the thruster flow circuit and the cathode flow circuit are modeled for the xenon flow, but the electrical and plasma transients are of secondary importance at the system level.

Another basic function of importance was to estimate the heating behavior of the cathode. This particular goal is a challenge facing simulations of Hall thrusters. ${ }^{4}$ The plasma interactions within the emitter insert are particularly complex and contain extreme temperature, density and potential gradients within very short distances. ${ }^{5}$ It would be ideal to be able to develop an algorithm for this feature, but the complexity and steep gradients make this task challenging and requires a more empirical approach. ${ }^{6}$

\section{AEPS Model Architecture}

System performance models are essential elements of the design and development process and they must be structured to enable rapid evaluation of requirements, components and interfaces to minimize program disruptions. The AEPS model is being developed in Simulink ${ }^{\circledR}$ to take advantage of its time-based modeling capabilities and to use the more complex mathematical processing features of Matlab ${ }^{\circledR}$. While time dependent, the model is a 0-D style model in order to function within a useful execution time such as minutes rather than hours or days.

Simulink ${ }^{\circledR}$ employs a number of solvers, but for this work the standard ode45 was chosen, as it has been proven to be a reliable solver with good results for most time-dependent simulation problems. ${ }^{7}$ Matlab ${ }^{\circledR}$ also offers many highly flexible options for data I/O. For this work, most data is stored as structures, which keeps the model well-organized, easy to read and maintainable. It also has extensive Hardware and Software In the Loop (HIL/SIL) capability, which may be useful in the future if such a capability is found to be necessary. 
The first step was to develop a system architecture identifying all of the interactions and connections. Figure 2 illustrates the basic flow of electrical, fluid, telemetry and plasma in basic schematic form. Each element model also includes the internal interfaces and interactions appropriate for the element. As can be seen there are a number of interactions between each of the major components. The interactions are electrical, fluidic, thermal and plasma based.

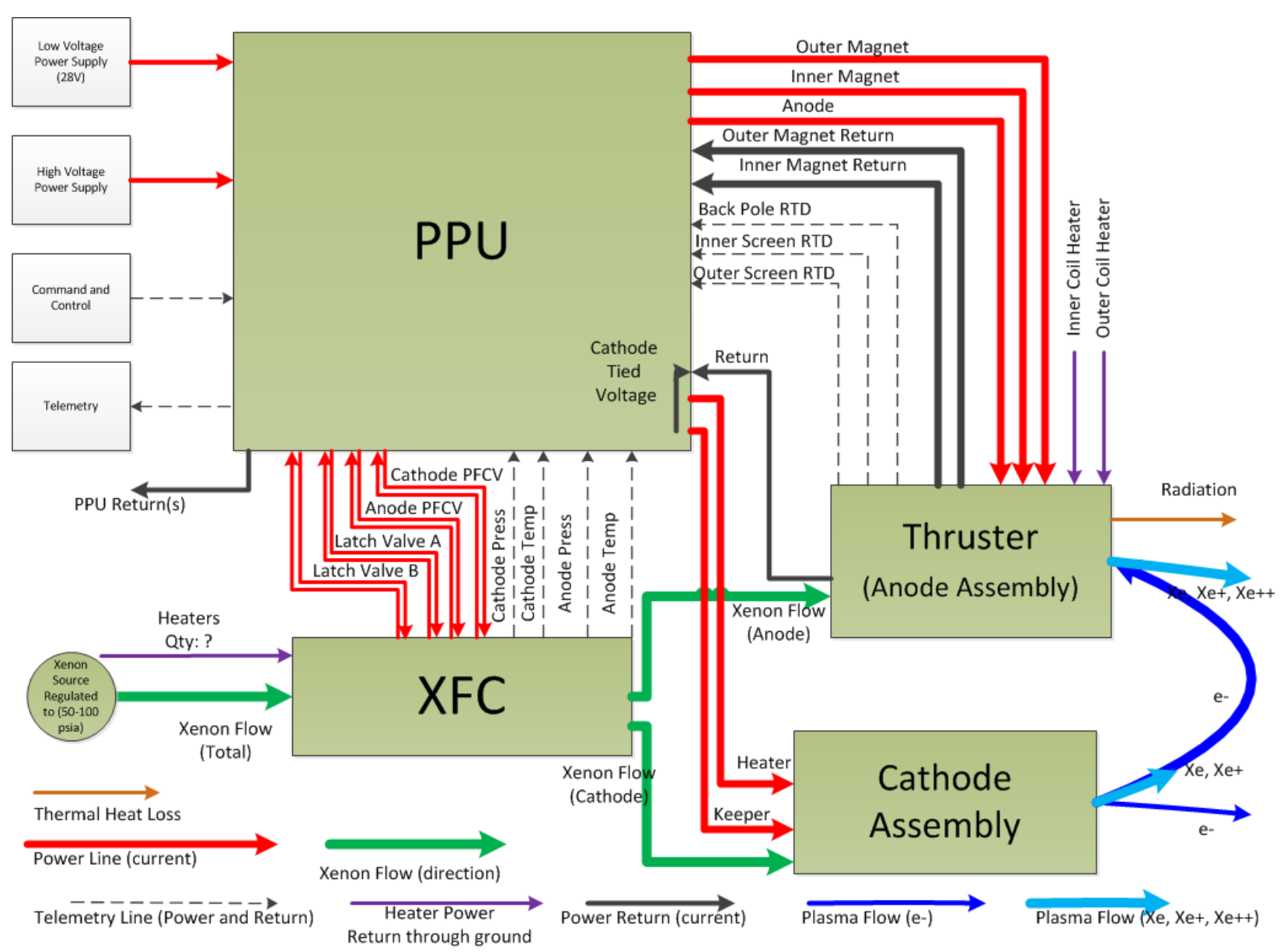

Figure 2. Advanced Electric Propulsion System Architecture.

When selecting a modeling architecture a number of factors play into how the problem is worked. For AEPS, it was observed that the system was both an electronic circuit and a fluid dynamic circuit and that those time scales are very different but closely related. The XFC component is generally a straightforward fluid dynamic set of calculations with mostly telemetry and flow commands returned in a similar time frame as the fluid behavior.

Meanwhile, the HT is more complex with interactions between the fluid dynamic time frame and the much faster electrical response time frame. The faster time frame and its interaction with the slower time frame provides for some of the more interesting behavioral symptoms of unsteady operation observed in Hall thrusters, such as the breathing mode.

The PPU behavior is on the faster electronic time frame, and the commands have to be slowed down through the use of appropriate gains to operate with the XFC and the HT. Internally, the PPU processes commands much faster than the physical flow can respond, but on time scales that could affect the plasmas by creating a feedback loop between the plasma natural frequencies and control unit.

In an ideal model, all of the time scales would be incorporated and addressed. For the AEPS system model the fluid dynamic portions of the system are relatively straightforward to model and of primary importance. However, some of the plasma dynamic behaviors are exceedingly complex and have to be approximated at this stage of development. The plasma dynamics approximations result in making the model less accurate at the frequencies associated with the electrical and plasma dynamic thruster time scales meaning that features such as the breathing 
mode will not be captured. It also means that plasma specific properties such as anomalous transport and nonequilibrium ionization are not modeled.

\section{AEPS Model and Major Component Description}

The AEPS system model is composed of the three primary elements: the PPU, the XFC and the HT. Each element has sub modules used to calculate the behavior of the element overall. The actual connections between the components in the simulation are more intricate than what is shown in the simplified system schematic in Figure 2. The schematic in Figure 3 shows all of the connections required to make the interactive AEPS system model. The additional connections relative to Figure 2 replicate the telemetry as well as the two way communication through an electrical connection or fluid or plasma volume, which requires two connections in a Simulink® simulation.

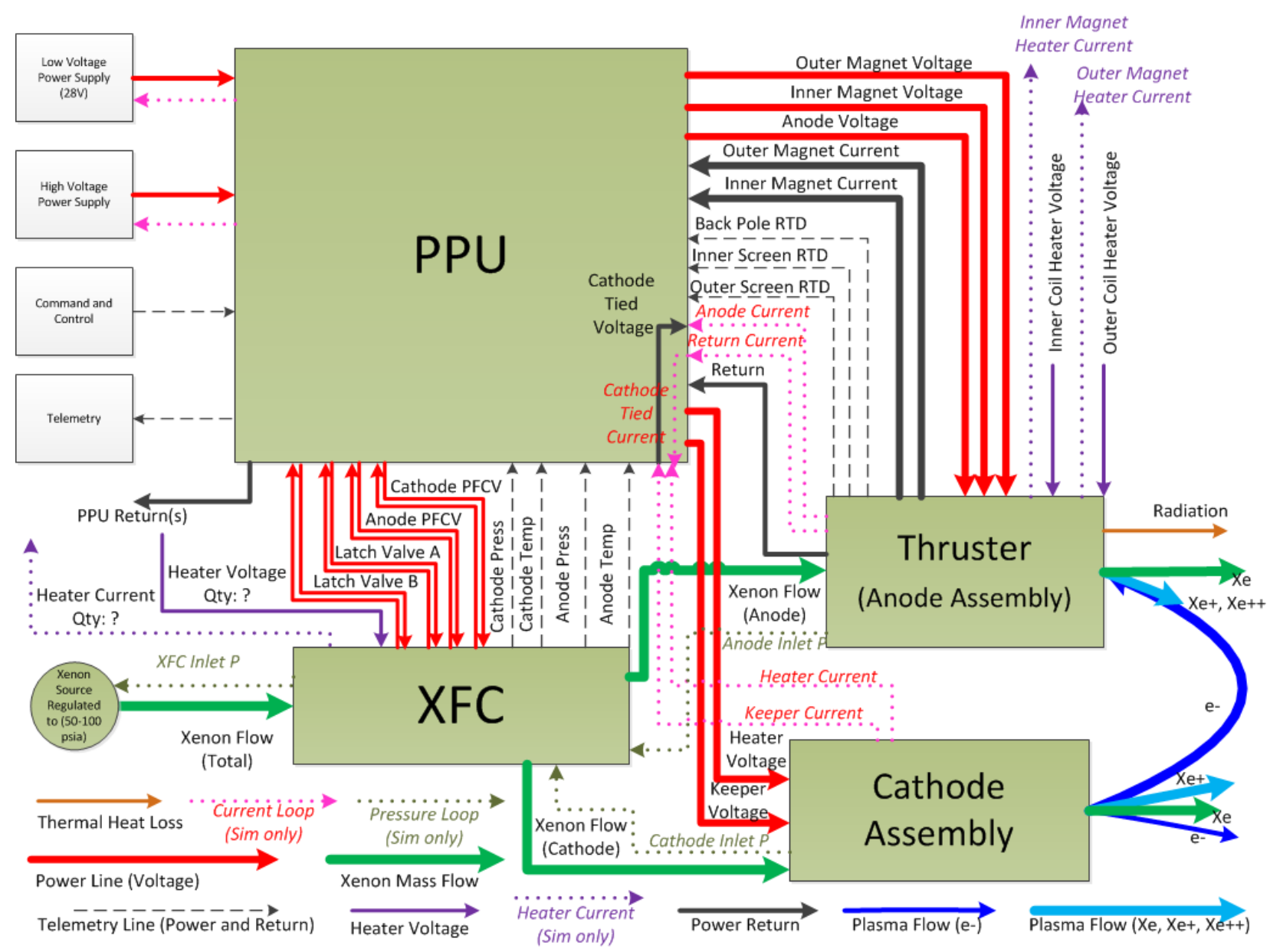

Figure 3. Advanced Electric Propulsion System Model Schematic with Feedback Loops.

\section{A. Power Processing Unit (PPU)}

The Power Processing Unit consists primarily of a bank of power supplies and associated control systems to provide both high and low power to the HT and the XFC. The PPU provides voltage input to the XFC to control the mass flow rate in order to maintain the overall current for the thruster. A common power supply module is used for all ten supply models. The six discharge supplies are bridged in order to maintain sufficient discharge voltage. The PPU controls the voltage applied to the HT and XFC in order to maintain the desired current and accepts the telemetry responses from the sub components. The current error drives the control loops to generate voltage commands for the XFC and most of the HT. For the HT anode assembly, the PPU sets a commanded voltage and then adjusts the mass 
flow rate to maintain a desired current. Gains for the control loops have not been established as of the time of this writing, but are in process. Figure 4 shows the Simulink module for the top level of the PPU.

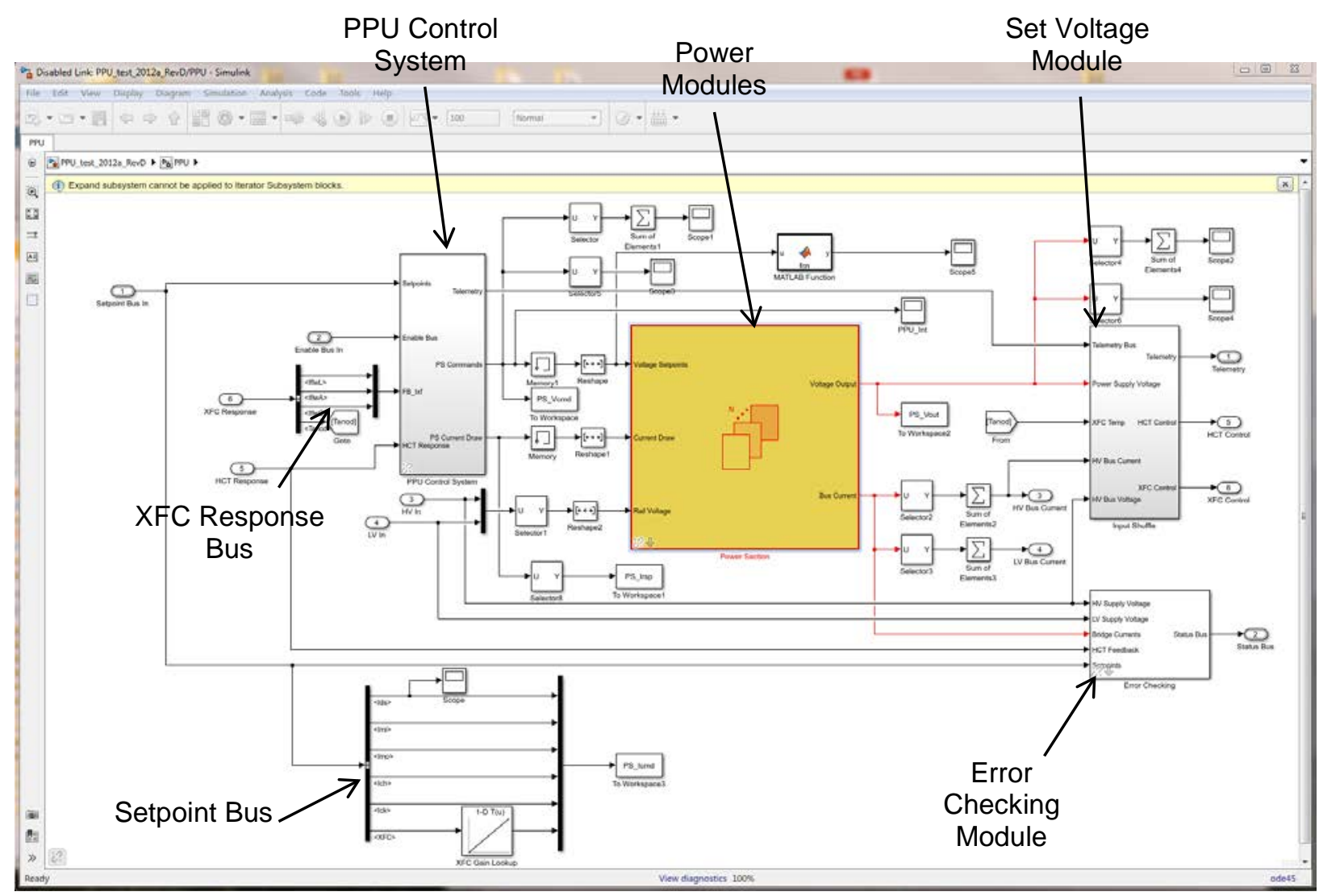

Figure 4. Simulink ${ }^{\circledR}$ System Model of the Power Processing Unit Component.

\section{B. Xenon Flow Controller (XFC)}

The Xenon Flow Controller (XFC) delivers a controlled flow rate of xenon propellant from a regulated xenon source such as a high pressure tank with a regulator to the thruster, responding to commands given by the PPU, which in turn responds to telemetry from the XFC. The XFC for the AEPS is supplied by VACCO. The AEPS XFC subsystem model follows methodology originally developed in-house at Aerojet Rocketdyne for modeling the similar but smaller XFC for the Aerojet Rocketdyne XR-5 Hall thruster. ${ }^{8}$

Figure 5 shows a conceptual schematic of the major components of the XFC. Regulated (50-100 psia) xenon flow enters the XFC from the left side of the schematic, passing through a filter on its way to a high pressure micro latch valve, which isolates the storage tank from the rest of the propulsion system. Fluid flow rate to each propellant plumbing leg (cathode and anode) is controlled by piezoelectric proportional flow control valves located downstream of the latch valve. These valves respond to voltage commands from the PPU, and require very little electrical power to operate. Pressure and temperature are measured upstream of sonic metering orifices, and transmitted back to the PPU to enable closed-loop flow control, enabling consistent measurement of the propellant flow rate to each leg. For the off-nominal condition of a regulator failure that would result in up to 3000 psia xenon entering the XFC, the differential pressure across the latch valve in the XFC can be very high, so electric heaters at the latch valve heat the propellant to prevent Joule-Thomson cooling and possibly freezing of the propellant downstream of the latch valve.

American Institute of Aeronautics and Astronautics 


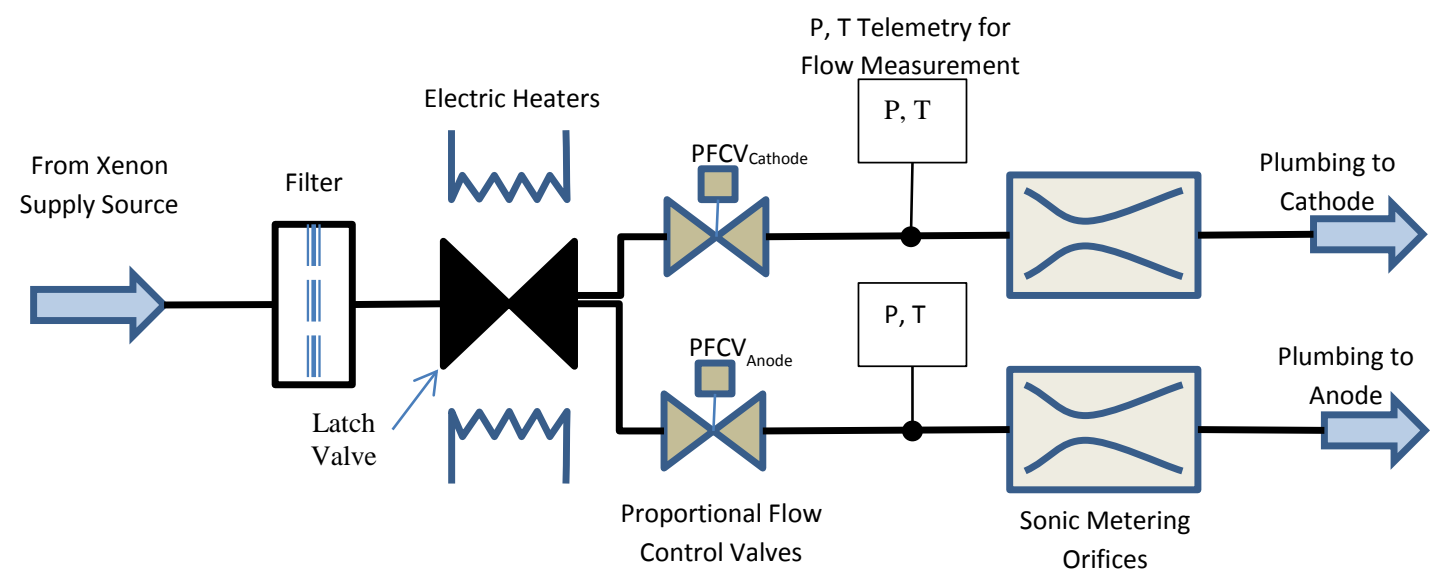

Figure 5. Xenon Flow Controller (XFC) Schematic.

The XFC model simulates both the XFC and plumbing connecting between the XFC and the HT. The AEPS XFC model follows methodology originally developed in-house at Aerojet Rocketdyne for modeling the response of an XFC for the Aerojet Rocketdyne XR-5 Hall thruster, with some modifications to account for real-fluid effects. ${ }^{8}$ Although never published, the Aerojet Rocketdyne model was validated against the XR-5 data. The original XR-5 model was developed in Mathcad ${ }^{\circledR}$ and includes simple models for the PPU and the XR-5 Hall thruster. The Simulink ${ }^{\circledR}$ AEPS model uses the algorithms from the XR- 5 model to simulate the XFC and associated plumbing, but has expanded fidelity for the thruster. The Simulink ${ }^{\circledR}$ model consists of individual blocks that each model a basic component of the XFC assembly, including fluid control volumes, valves, and long tubing lengths.

The Fluid Control Volume (FCV) block applies conservation of mass and enthalpy in a control volume, tracking inflows and outflows of Xenon to account for changes in thermodynamic state of the Xenon in the FCV. For a pure fluid of known composition, the thermodynamic state is completely defined once two thermodynamic state variables are known, so lookup tables can be used to convert the computed density and internal enthalpy into pressure and temperature. The properties used to populate the thermodynamic lookup tables were generated using the NIST REFPROP database software tool, which tabulates thermodynamic and transport properties for many fluids, including Xenon. ${ }^{9}$ Since REFPROP includes non-ideal fluid properties, the methodology can account for Joule-Thomson cooling in the fluid as it expands from the high pressure storage tank to the low pressure plumbing downstream of the XFC, as well as adjust to enthalpy addition via electric heating. ${ }^{9}$ The FCV blocks are used in the internal volume the $\mathrm{XFC}$ as well as within the plumbing.

The valve block uses the known thermodynamic states in the FCVs upstream and downstream of the valve, as well as valve flow tables, to calculate the flow rate through the valve. The valve flow tables are determined by calibration testing. The applicable range of operation of the flow tables can be extended by applying theoretical relations governing gaseous flow through an orifice, such as for situations when the flow changes from choked to unchoked flow. Valve hysteresis (where the flow rate at a given valve voltage command signal differs between valve opening and closing) is modeled using the backlash module included in Simulink ${ }^{\circledR}$.

Since the plumbing connecting the XFC to the HT may include long, thin tubing, dependent upon application, the XFC model includes blocks to estimate the pressure losses along the tubes due to fluid friction. These losses are estimated using theoretical equations for adiabatic flow of a perfect, viscous gas in a constant-area tube. ${ }^{9}$ The plumbing line blocks combine the FCV and pipe flow blocks.

Figure 6 shows a screen capture of an early version of the Simulink ${ }^{\circledR}$ model of the XFC and plumbing. The latch and proportional flow control valves (PFCVs) are modeled using the valve blocks discussed above. The inter-valve volume block uses the FCV methodology to track the fluid thermodynamic state. The plumbing is divided into multiple segments and an insulated isolator, to enable tracking pressure along the plumbing lines. Each of these blocks is modeled by pairing a FCV and pipe flow block together. 


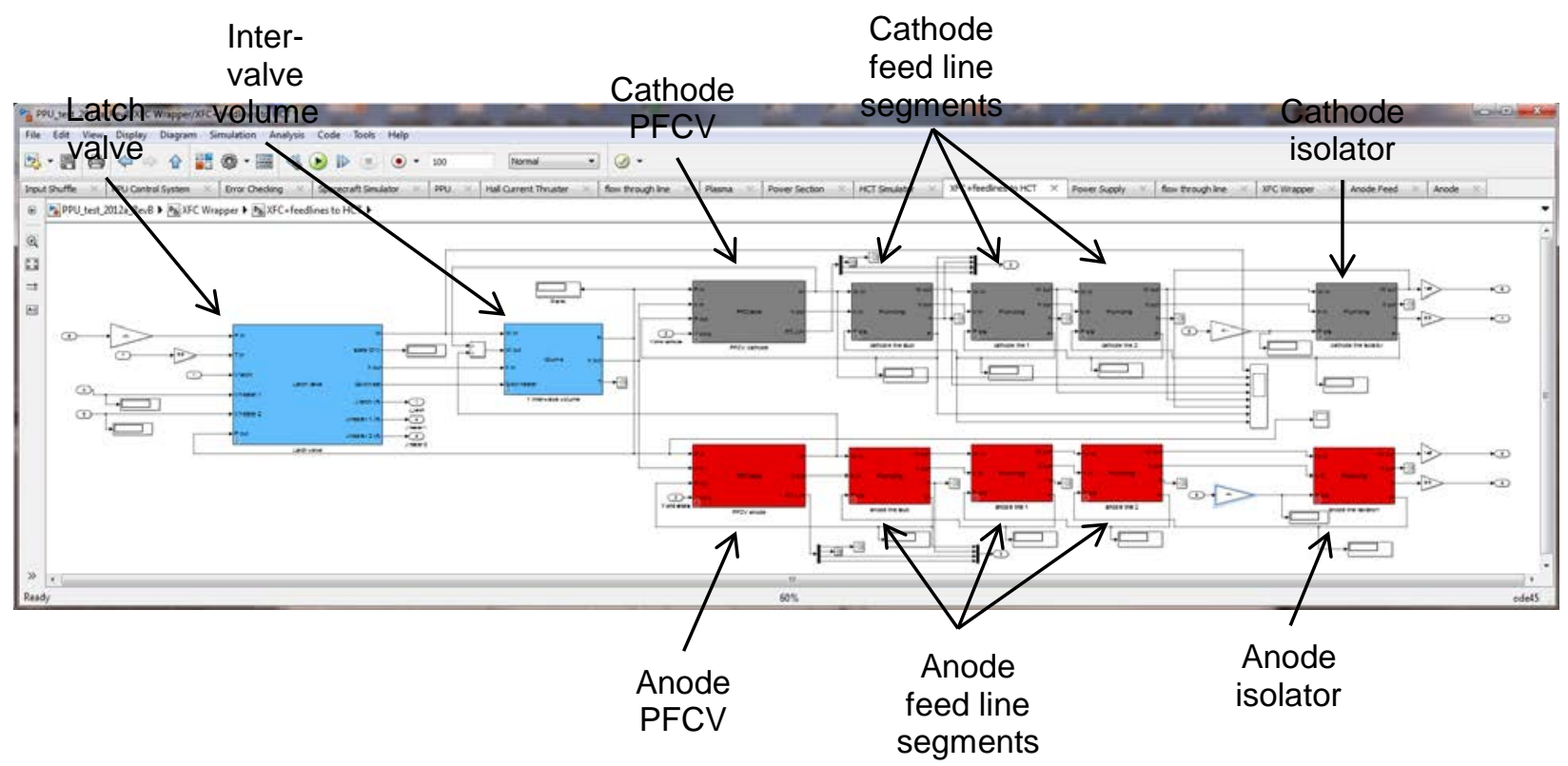

Figure 6. Simulink ${ }^{\circledR}$ System Model of Xenon Flow Control and Plumbing Subsystem.

The interfaces for the XFC model mirror the interfaces for the physical article. A schematic of the system, showing the interfaces with the other component models, is shown in Figure 7. The dashed box encloses the components simulated by the XFC subsystem model, including the XFC and the associated plumbing to the thruster. The arrows indicate data flow into and out of the XFC model. An arrow into the box indicates input to the XFC model, while an arrow pointing out indicates output from the XFC model.

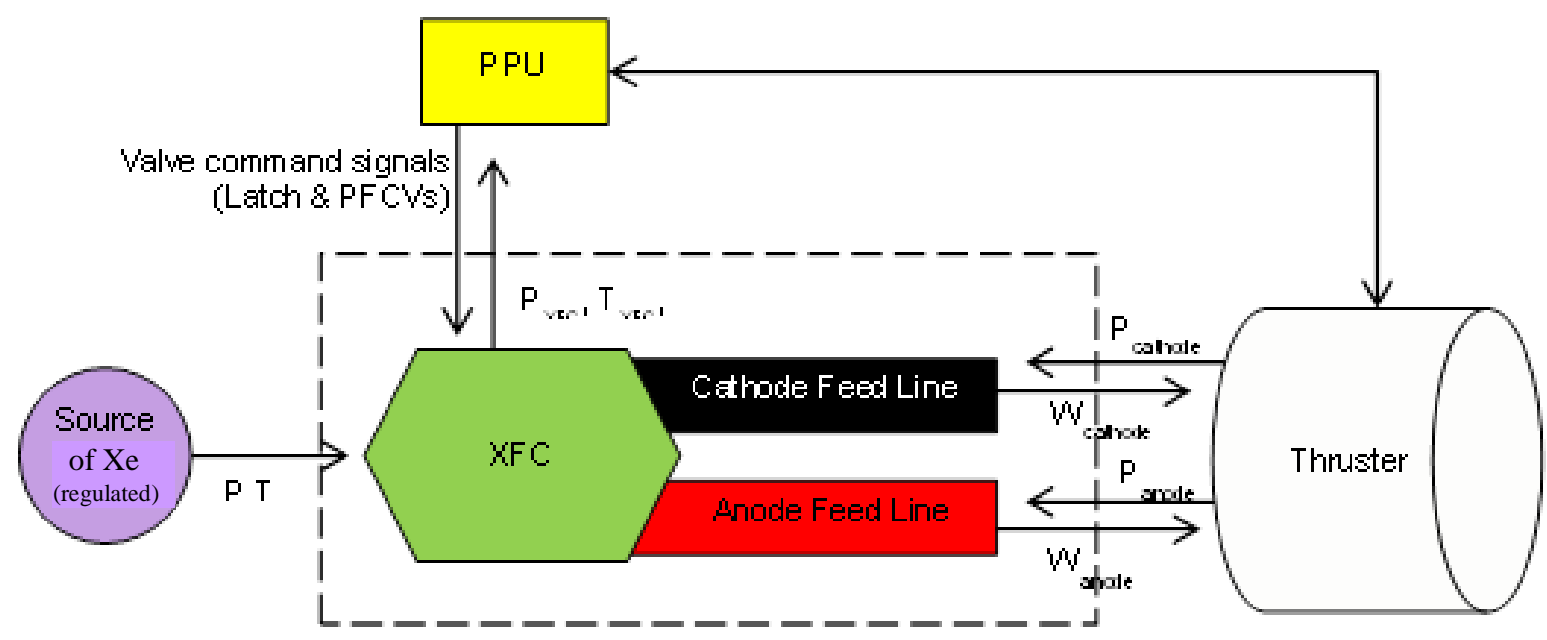

Figure 7. Xenon Flow Controller Subsystem Model Interface Schematic.

The inlet boundary condition for the XFC is the pressure and temperature of the xenon downstream of the spacecraft regulator that regulates the xenon stored in the propellant tank at up to 3,000 psia to a range of 50-100 psia entering the XFC. The interface with the PPU includes electrical voltage commands from the PPU to the XFC, and pressure and temperature telemetry from the XFC transmitted back to the PPU. The interface with the thruster includes the flow rate of propellant delivered to the thruster and the fluid pressure at the anode and cathode (used as an exit boundary condition for the XFC model). Table 1 lists the inputs and outputs from the XFC model and their interface. Other model input parameters are set by an input script that is run from the Matlab ${ }^{\circledR}$ workspace prior to running the system model.

American Institute of Aeronautics and Astronautics 
Table 1. Xenon Flow Controller Model Outputs

\begin{tabular}{|c|c||c|}
\hline Input Parameter & Output Parameter & Connecting Module \\
\hline \hline Inlet Pressure & & Spacecraft \\
\hline Inlet Temperature & & Spacecraft \\
\hline Cathode Pressure & Cathode Mass Flow Rate & HT \\
\hline Anode Pressure & Anode Mass Flow Rate & HT \\
\hline Cathode Voltage & Cathode Current & PPU \\
\hline Anode Voltage & Anode Current & PPU \\
\hline Latch Valve Voltage & Latch Valve Current & PPU \\
\hline & Cathode Mass Flow Rate & PPU \\
\hline & Anode Mass Flow Rate &
\end{tabular}

\section{Hall Thruster (HT)}

The Hall Thruster (HT) is the propulsive device in the AEPS. The thruster itself consists of two primary elements: the cathode and the magnet/anode features, each with multiple connections to the PPU, XFC and each other. The thruster produces thrust by generating plasma in the discharge channel and then electrically accelerating that plasma in the axial direction. The physics describing how Hall thrusters work is beyond the scope of this paper, but the interested reader is encouraged to refer to Goebel and Katz. ${ }^{10}$

The cathode is the first element of interest in the HT. The cathode is a small hollow tube with an inlet for xenon flow at one end and an orifice to control the pressure within the cathode at the other end. The flow in the cathode is modeled as Poiseuelle flow modified for compressible gas with some complexities added. ${ }^{10}$ The cathode has a thermionic emission insert in it at the end near the orifice. The thermionic insert is heated by an external heater to generate electrons during the startup procedure. Electron bombardment of the xenon flow creates plasma near the orifice. ${ }^{11}$ The electrons are emitted as a function of insert temperature, which in turn is further heated by xenon ions hitting the insert surface. The insert eventually achieves a thermal balance between the ion heating and plasma generation from electron emission, and the cathode no longer requires the heater to be active.

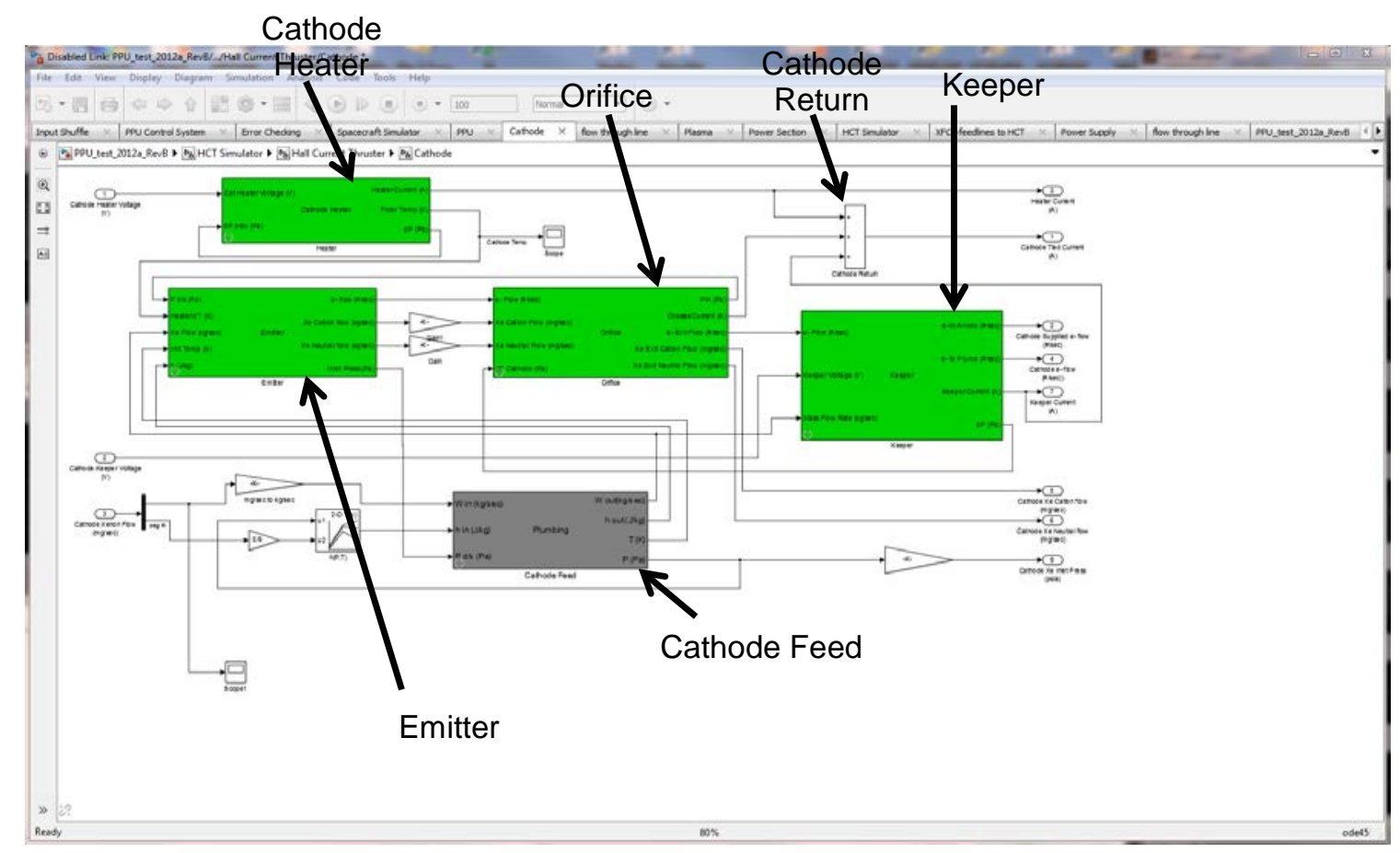

Figure 8. Cathode Assembly Simulink ${ }^{\circledR}$ Module.

American Institute of Aeronautics and Astronautics 
The cathode also has a small positively charged anode (not to be confused with the HT anode located at the base of the discharge channel) called the keeper, located just downstream of the orifice. The keeper is used to "ignite" the plasma by drawing out some of the electrons in the plasma through the application of a high voltage to the keeper. The electrons pull with them a few positive xenon ions due to the principle of quasi neutrality, thus establishing a flow from the interior of the cathode to the exterior. After thruster ignition, the keeper is turned off, but the electrical circuit has been established and flow continues. The circuit and flow interactions are captured in the Matlab ${ }^{\circledR}$ screen shot of the cathode module shown in Figure 8.

Within the cathode, there are steep density, temperature and species concentration gradients with respect to axial position. Slight variations can have rather dramatic effects on the cathode and can change the potential and current from the cathode. An extreme shift could cause the cathode to shift from spot-mode to plume-mode operation, which could have a major effect on the system life. Due to the sudden and sharp transitions that can occur, the system could experience transitions which look like turning on and off multiple switches or inputting random discontinuities into the simulation resulting in an unstable model. For the AEPS simulation, the threshold for plume mode and other step changes in the plasma behavior will not be used initially due to the complexity of the physics and the challenges with the simulation. However, the potential of performance discontinuities will be evaluated and monitored.

The other major aspect of the thruster is the magnet and anode behaviors. The relationship between discharge voltage, magnetic field strength, flow rate and discharge current are illustrated in Figure 9. As can be seen, the effect of variation in magnetic field strength and discharge voltage are secondary in comparison to the mass flow rate with respect to the discharge current. Thus, a relatively accurate map (flat planes) with errors $<+/-5 \%$ of full scale can be made of the four parameters.

The plasma field generated by the mass flow, discharge current and magnetic field in the channel drives the operation of the thruster. As electrons are emitted by the cathode, a fraction of them are attracted to the anode at the base of the discharge channel. The electron flow from the cathode to the anode is often called leakage current. ${ }^{10}$ The rest of the electrons from the cathode balance out the xenon ions from the thruster. Electrons entering the channel are trapped in the magnetic field generated by the electromagnet

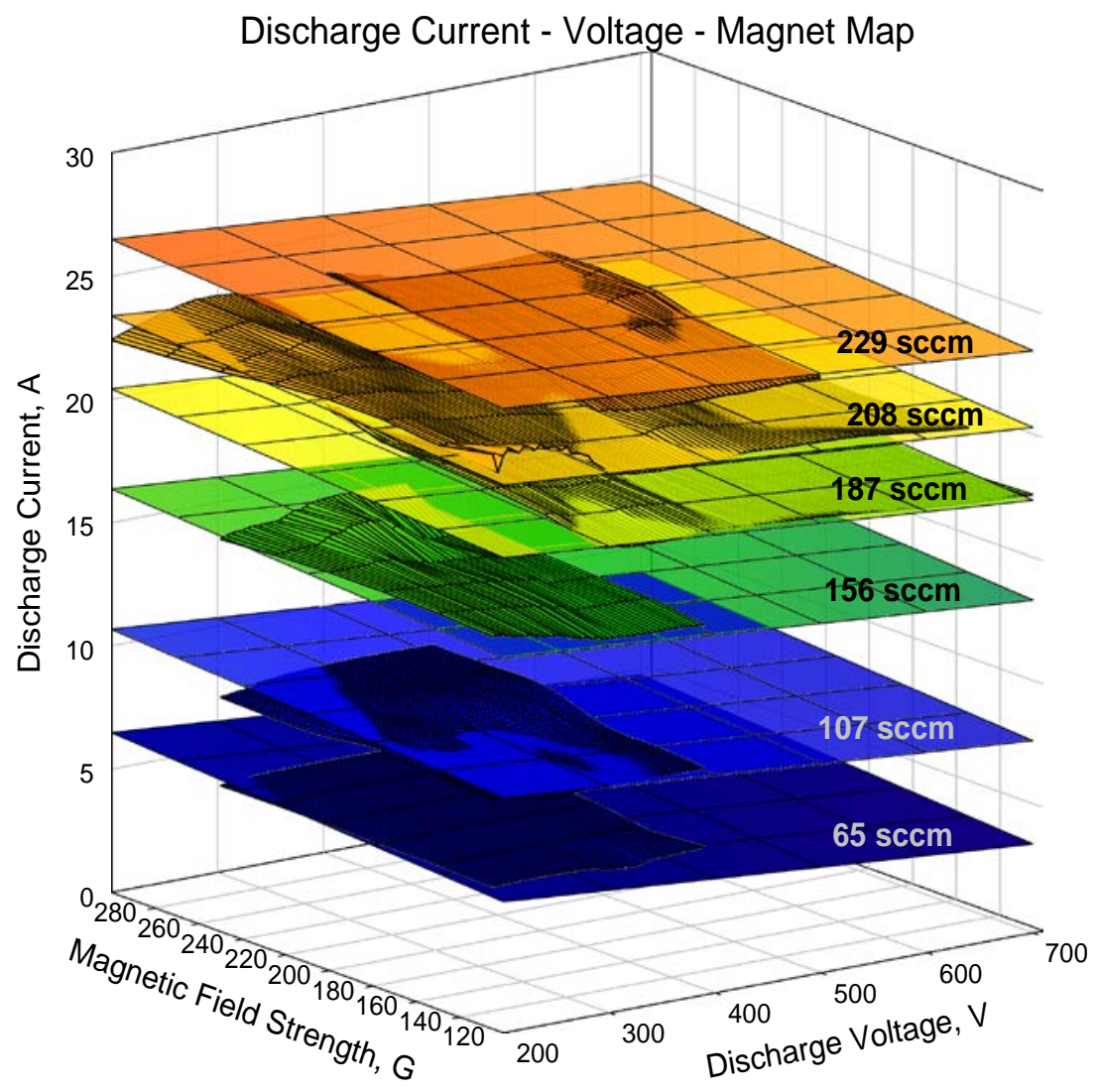

Figure 9. Advanced Electric Propulsion System Voltage-MagnetCurrent Map. created by the two wound inductors and magnetic material core. The trapped electrons form a sheet or region of highly energetic electrons through which the main xenon flow passes. Nearly all of the xenon is ionized as it passes through the highly energized electron region. As the electrons collide with the xenon some of the electrons drop out of the magnetic field and are attracted to the anode, thus completing the electrical circuit from the cathode to the anode to the chassis. Since the cathode is also tied to the chassis, the electrons are allowed to recycle back to the cathode. Figure 10 shows the Matlab ${ }^{\circledR}$ module used to model the thruster. 


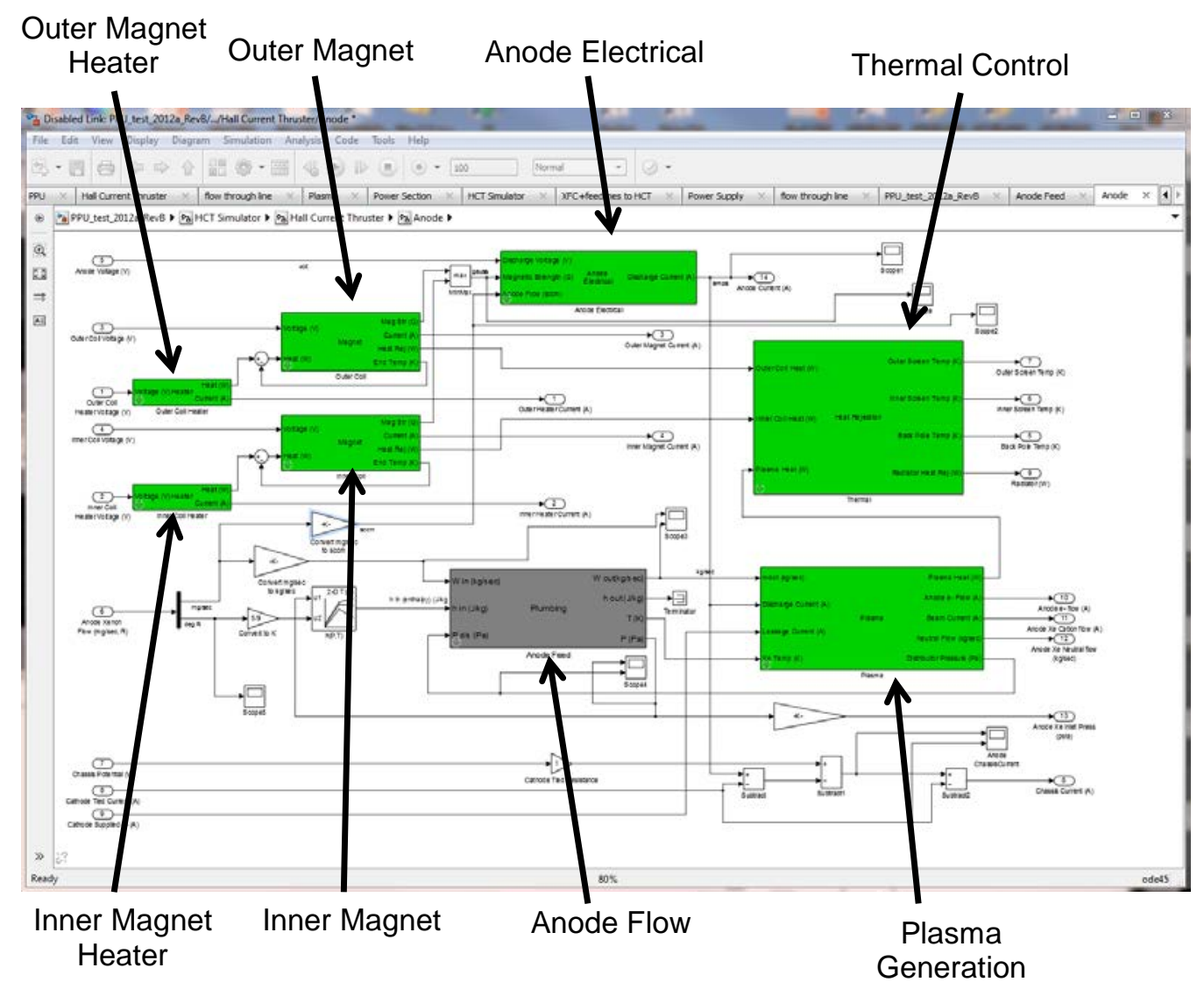

Figure 10. Hall Current Thruster Simulink ${ }^{\circledR}$ Module.

The inputs and outputs for the HT module are numerous and relate to both the PPU and the XFC. In many cases, inputs and outputs are related through the same physical interface. For example, the module accepts a voltage input for a heater and returns the current based on the resistivity and temperature of the heater, but in reality both the voltage and current are sensed through the same wire. This should not be confused with the control logic which actually adjusts voltage to maintain an input current. Table 2 below is set up to illustrate the relationship between primary outputs and inputs for the simulation. In addition to the outputs listed, the module also provides temperature estimates in a number of locations, radiation from the thruster and a breakdown of the cathode and anode plumes. The details in the plumes are rather rudimentary at the time of writing. 
Table 2. Hall Thruster Module Inputs and Outputs.

\begin{tabular}{|c|c|c|}
\hline \multicolumn{1}{|c|}{ Input Parameter } & Output Parameter & Connecting Module \\
\hline \hline Discharge Voltage & Discharge Current & PPU \\
\hline Cathode Heater Voltage & Cathode Heater Current & PPU \\
\hline Cathode Keeper Voltage & Cathode Keeper Current & PPU \\
\hline Inner Pole Voltage & Inner Pole Current & PPU \\
\hline Outer Pole Voltage & Outer Pole Current & XFC \\
\hline Cathode Xenon Flowrate & Cathode Inlet Pressure & XFC \\
\hline Anode Xenon Flowrate & Anode Inlet Pressure & PPU \\
\hline Chassis Potential & Chassis Current & PPU \\
\hline Outer Coil Heater Voltage & Outer Coil Heater Current & PPU \\
\hline Inner Coil Heater Voltage & Inner Coil Heater Current & \\
\hline
\end{tabular}

\section{Correlation with NASA Test Data}

Validation of the system model is an important step in establishing the usefulness of the simulation. The validation process starts with establishing a set of data to match to and a set of data to test against. The model has a number of magnetic and thermal trends which can be matched in a straightforward manner by adjusting one constant or another. Similarly, the fluid dynamics can be matched with a little more effort. However, the plasma physics are more complicated to adjust in order to match measured test data due to the number of assumptions made in using empirical data. There are three sets of data from NASA testing that will be used in the validation process. Then early system testing will be used to anchor the model to the flight configuration.

Existing test data can be compared to performance profiles generated by the transient simulation tool to anchor and validate the model. The following figures illustrate some experimental measurements taken during startup tests of the thruster. Figure 11 shows the applied voltage to key components of the thruster and Figure 12 displays the corresponding electrical currents.

Figure 13 plots the propellant mass flow through the anode and cathode, and Figure 14 shows the associated thrust measurement. Some of the voltage and mass flow data shows high frequency oscillations and/or data dropouts, which may be smoothed out as needed to provide appropriate input data for the model. The transient model responses will be adjusted as necessary to match the output test data behavior as closely as possible.

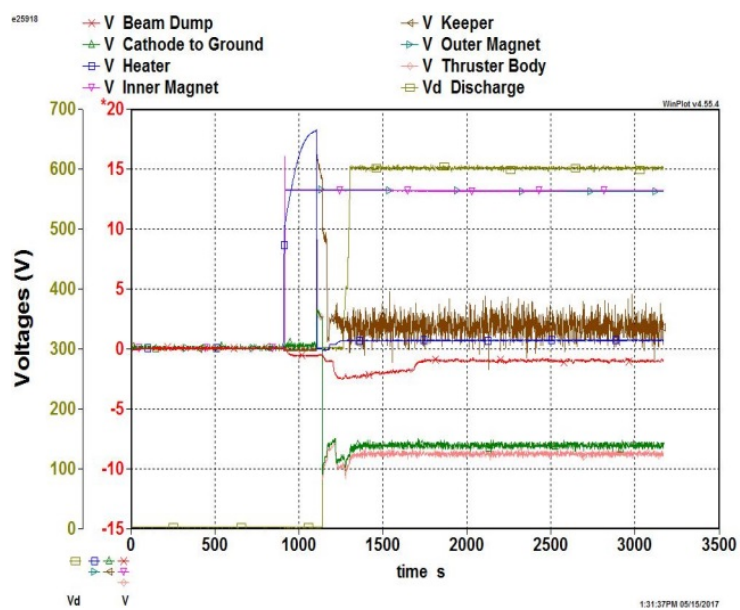

Figure 11. Test Development Unit Thruster Start Voltage Data.

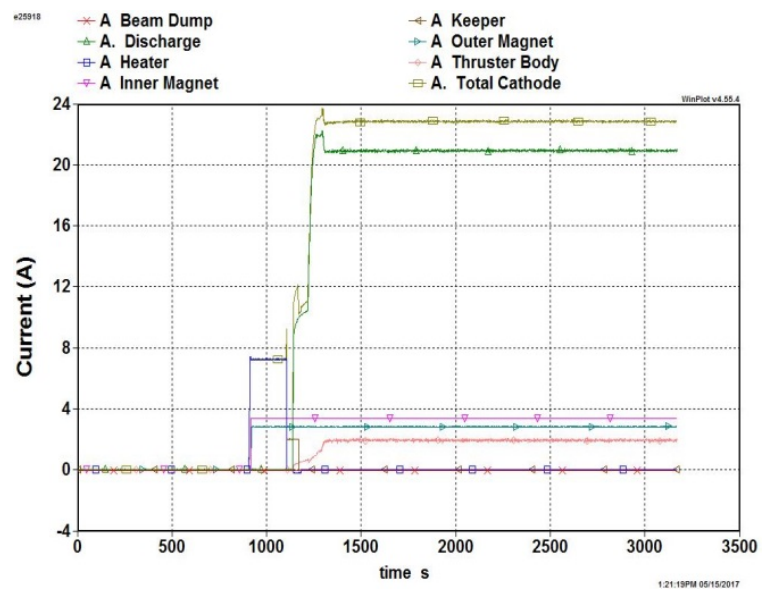

Figure 12. Test Development Unit Thruster Start Current Data.

American Institute of Aeronautics and Astronautics 


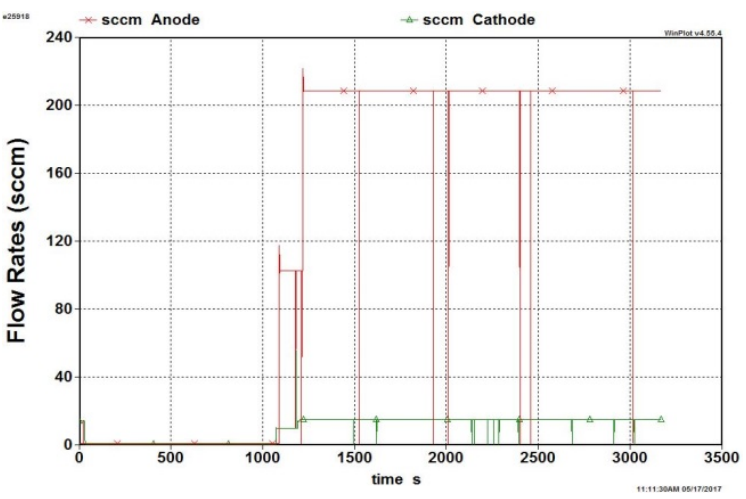

Figure 13. Test Development Unit Thruster Mass Flow Start Data.

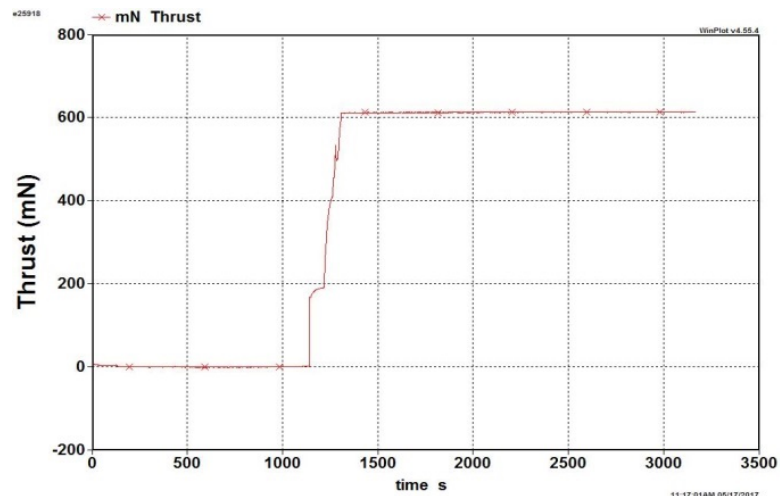

Figure 14. Test Development Unit Thruster Start Thrust Data.

The AEPS program is intended to develop a PPU and its control logic prior to the first time the full system with the flight like XFC, PPU and HT will be tested. Thus, the intent is to use the NASA gathered data (shown in Figure 11 to Figure 14) and supplier data to put together the full system model. Then, the model will be anchored to an early system level test using similar hardware to the flight system, but not identical. The simulation will be adjusted, if necessary, to match the final configuration.

\section{Future Effort and Uses for the AEPS Model}

Several capabilities were initially deemed to be worthwhile for the system model. However, during the development of the model it was determined that several of the capabilities were either beyond the current understanding of the physics by the community or would result in an overly complex simulation. The more complex phenomena are being placed on the list of potential improvements for the model and will be addressed as opportunities present themselves in the future.

The model was deliberately built in a modular format in order to support possible future expansion as more information/understanding is obtained in one area or another. Each module can be upgraded or modified or even replaced as improvements in assumptions or understanding allows. The model can be upgraded as time goes on to provide improved capability.

Additionally, the simulation was developed with all of the key parameters defined in configuration files. Changing the type of XFC, the size of feed line, the magnet size, etc. are all accomplished through text files and do not require any adjustment to the construction of the model or need to go into the model to change a value. Thus, the model is being developed to be flexible enough to simulate multiple Hall thruster systems.

The basic capabilities are being incorporated into the current system model. These capabilities include fluid flow response and performance for the xenon flow control for both the cathode flow and the thruster flow. The flow principles are modeled based on first principles and provide methods for evaluating start transients, throttle transients and low frequency stability. Ultimately, this will provide insight into the stability of the system and how sensitive it will be to line length changes when AEPS goes to a flight configuration.

However, the current lack of plasma dynamics on a 0-D or 1-D basis does limit the utility of the model. If greater understanding is gained in those areas where simple lumped parameters can be used to model their behavior, then additional improvements can be made to the simulation. For example, it might become possible to relate cathode leakage current to the operating condition of the HT, which would provide insight into the system level performance and how the plasma frequency can influence high frequency stability. At that point, the model may become predictive enough to evaluate characteristic changes which could improve thrust, efficiency, operability and the life of thrusters.

\section{Conclusion}

The AEPS system model is a simplified model being developed at Aerojet Rocketdyne to aid in the development of the full AEPS system. The model will replicate the fluid dynamic and overall electrical behavior of the system and 
its response to control inputs. However, the initial usage of the system model will not include the plasma dynamic characteristics needed to make the simulation a true physics based model of the AEPS system. Instead empirical approximations will be used for many of the faster electronic and plasma based mechanism. Thus, the simulation will adequately evaluate the lower frequency behavior of the system such as the fluid dynamic trends, but will not be able to resolve the higher frequency behavior of the system.

With this simulation, it will be possible to model different thruster, PPU, XFC combinations as well as evaluate some of the changes for each use of the system such as propellant line length, operating pressure and startup differences. As time progresses, it is hoped that additional features can be added to the model to start capturing various operating characteristics at the higher frequencies. Given the depth of the complexities associated with the plasma dynamics, especially in the cathode, it might be some time before a first principles simulation resolves most of the plasma and electronic behaviors. However, the platform for working toward that goal is being developed.

\section{Acknowledgments}

The authors would like to acknowledge the support of NASA for the opportunity to develop the AEPS system model as well as the Jet Propulsion Laboratory for their ongoing support of AEPS. The collaborative effort of this program with our teammates from NASA GRC and JPL has brought together the electric propulsion community working toward a common goal of producing a system that will enable the advancement of many commercial and exploration goals.

\section{References}

${ }^{1}$ Hoskins, W.A., Cassady, R.J., Morgan, O., Myers, R.M., Wilson, F., King, D.Q., and deGrys, K., "30 Years of Electric Propulsion Flight Experience at Aerojet Rocketdyne,” 33 ${ }^{\text {rd }}$ International Electric Propulsion Conference, IEPC-2013-439, Washington, D.C., Oct. 6-10, 2013.

${ }^{2}$ Hofer, R., Polk, J., Sekerak, M., Mikellides, I., Kamhawi, H., Verhey, T., Herman, D., Williams, G., “The 12.5 kW Hall Effect Rocket with Magnetic Shielding (HERMeS) for the Asteroid Redirect Robotic Mission,” 52 ${ }^{\text {nd }}$ AIAA/SAE/ASEE Joint Propulsion Conference, AIAA 2016-4825, Salt Lake City, UT., July 25-27, 2016.

${ }^{3}$ Katz, I., Lopez Ortega, A., Goebel, D., Sekerak, M., Hofer, R., and Jorns, B., "Performance and Facility Background Pressure Characterization Tests of NASA's 12.5-kW Hall Effect Rocket with Magnetic Shielding Thruster," in 14th Spacecraft Charging Technology Conference, ESA/ESTEC,, Noordwijk, NL USA, 2016.

${ }^{4}$ Domonkos, M. T., “A particle and Energy Balance Model of the Orificed Hollow Cathode,” $38^{\text {th }}$ AIAA/ASME/SAE/ASEE Joint Propulsion Conference \& Exhibit, AIAA 2002-4240, Indianapolis, IN., July 7-10, 2002.

${ }^{5}$ Katz, I., Anderson, J. R., Polk, J. E., Brophy, J. R., “One-Dimensional Hollow Cathode Model,” Journal of Propulsion and Power, Vol. 19, No. 4, July-August 2003, pg. 595-600..

${ }^{6}$ Mandell, M. J., Katz, I., “Theory of Hollow Cathode Operation in Spot and Plume Modes,” 30 ${ }^{\text {th }}$ AIAA/ASME/SAE/ASEE Joint Propulsion Conference, Indianapolis, IN., June 27-29, 1994.

${ }^{7}$ Wilber, J., “BAE Systems Proves the Advantages of Model-Based Design,” https://www.mathworks.com/company/ newsletters/articles/bae-systems-proves-the-advantages-of-model-based-design.html, MathWorks ${ }^{\circledR}, 2006$.

${ }^{8}$ Shapiro, A.H., The Dynamics and Thermodynamics of Compressible Fluid Flow, John Wiley \& Sons: New York, 1953, p. 163.

${ }^{9}$ Lemmon, E. W., Huber, M. L., McLinden, M. O., NIST Reference Fluid Thermodynamic and Transport PropertiesREFPROP, National Institute of Standards and Technology, U.S. Department of Commerce, Boulder, CO, April, 2013.

${ }^{10}$ Goebel, D. M., Katz, I., Fundamentals of Electric Propulsion: Ion and Hall Thrusters, Jet Propulsion Laboratory, California Institute of Technology, CA, 2008, Chaps. 3, 6, 7 and Appendices.

${ }^{11}$ Mikellides, I. G., Goebel, D. M., Snyder, J. S., Katz, I., "Neutralizer Hollow Cathode Simulations and Validation with Experiments," 45 th AIAA/ASME/SAE/ASEE Joint Propulsion Conference \& Exhibit, AIAA-2009-5196, Denver, CO, USA, 2009.

American Institute of Aeronautics and Astronautics 